STUDIEN ÜBER DIE REVOLUTION 


\section{STUDIEN ÜBER DIE REVOLUTION}

In Verbindung mit

\section{MANFRED KOSSOK}

Abdel Malek Audah, Kairo

Germain Ayache, Rabat

Ranuccio Bianchi-Bandinelli, Rom

József Bognar, Budapest

Georges Castellan, Vincennes

Emanuel Condurachi, Bukarest

E. C. Fry, Canberra

Thomas Hodgkin, Oxford

Kyösti Julku, Oulu

Hilde Koplenig, Wien

Dimiter Kosev, Sofia

Ernst Werner, Leipzig

Djibril Tamsir Niane, Conakry

Francisco Posada, Bogota

Carlos M. Rama, Montevideo

Alfred Rufer, Bern

Rodolfo Stavenhagen, Mexiko, D. F.

Stefan Strelcyn, Warschau

S. I. Tjul'panov, Leningrad

Jujiro Uesugi, Tokio 


\section{STUDIEN \\ ÜBER DIE \\ REVOLUTION}

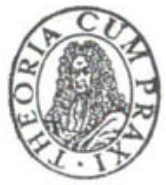

AKA DEMIE-VERLAG - BERLIN 1969 
Erschienen im Akademie-Verlag GmbH, 108 Berlin, Leipziger Str. 3-4 Copyright 1969 by Akademie-Verlag $\mathrm{GmbH}$

Lizenznummer: $202 \cdot 100 / 61 / 69$

Gesamtherstellung: VEB Druckhaus „Maxim Gorki“, 74 Altenburg

Bestellnummer: 5736 - ES 5 G 14 D 